Psychological Medicine, 1987, 17, 271-274

Printed in Great Britain

\title{
EDITORIAL
}

\section{Genetic latent structure models: implication for research on schizophrenia ${ }^{1}$}

Our thinking about the strategy of research on schizophrenia has turned in new directions because of a genetic model which we recently developed to account for some paradoxical data on smooth pursuit visual tracking. The new strategy, and the logic behind it, may be of interest to other investigators in psychiatric research.

A simple test of smooth pursuit eye movements revealed that about two-thirds of schizophrenic patients and about half their first-degree relatives show abnormal eye-tracking movements (Holzman, 1985). A series of studies demonstrated that these eye-movement disorders were not produced by inattention, mechanical interferences, or neuroleptic drugs. Although similar eye movement abnormalities are found in patients with major affective disorders, principally mania, the vast majority of those patients were being treated with lithium carbonate which alone among the antipsychotic drugs can produce pursuit abnormalities.

The abnormalities observed in schizophrenic patients consist of very frequent interruptions of pursuit movements by saccadic eye movements which appear as single pulses, double saccadic pulses, 'square-wave jerks', anticipatory saccades, and retrosaccades. The abnormality occurs when the eyes are driven by cortical mechanisms, as when following an object, but not when driven by brain stem or vestibular mechanisms, as in self-movement or the illusion of self-movement.

Two studies of monozygotic (MZ) and dizygotic (DZ) twins discordant for clinical schizophrenia showed that the MZ twins were almost perfectly concordant for smooth pursuit dysfunctions and the DZ twins were almost $50 \%$ concordant. A recent review of the principal studies of eye movement abnormalities in schizophrenia strongly suggests that these visual tracking abnormalities can illuminate the biology and particularly the genetics of schizophrenia (Holzman, 1985).

There is a simple observation which does not fit comfortably with the standard genetic models of either schizophrenia or smooth pursuit tracking: namely, schizophrenics with good tracking tend to have relatives with bad tracking, even when those relatives are not schizophrenic. For example, in 5 of 30 sets of dizygotic twins discordant for schizophrenia, the schizophrenic proband had good eye tracking but the healthy co-twin had impaired tracking (Holzman et al. 1977, 1980). A moment's reflection will show that the obvious models do not work. There seems at first to be no reason why the healthy first-degree relative should have bad tracking. Schizophrenia may cause bad tracking, but he is not schizophrenic; bad tracking may be inherited, but the proband to whom he is related had good tracking.

Our model attempts to account for this finding in the most straightforward fashion. We postulate that there is a latent trait which is not directly observable, but which can cause either schizophrenia, bad tracking, or both. It is the latent trait that is to be regarded as genetically transmitted, rather than the manifest traits of schizophrenia or bad tracking. Although the manifest traits may be said to be 'genetic' along with the latent trait, the idea behind the model is that the transmission pattern of the latent trait may be closer to that of a Mendelian gene with high penetrance than either of the manifest traits alone.

The latent trait may be regarded as a disease process in the brain, which can independently invade one region or another, and gives rise to different symptoms depending on which region or system is affected. Since bad tracking is much more common than schizophrenia, we postulate that the

\footnotetext{
1 Address for correspondence: Professor P. S. Holzman, Department of Psychology, Harvard University, William James Hall, 33 Kirkland Street, Cambridge, MA 02138, USA.
} 
disease process is more likely to invade the smooth pursuit system than the systems involved in schizophrenia.

The concept behind this model is actually quite old; its intellectual father is Pierre Marie (1873), who - in attempting to account for the similarities and differences between Friedreich's ataxia and the disease he named 'hereditary cerebellar ataxia' - suggested that these two syndromes might have a common origin:

I wonder whether the pathologic changes of hereditary cerebellar ataxia are not closer to Friedreich's ataxia than was apparent at first glance. The divergences between them are more a product of differences in symptoms than pathology. It is possible that both are parts of the same morbid process. This hereditary degenerative process as represented in the two conditions might initially affect similarly functioning but anatomically distinct nervous centers.

Quantitative details of the model have been published elsewhere (Matthysse $e t$ al. 1986). It accounts well for the available pedigree data on schizophrenia and smooth pursuit tracking.

Having worked out this model, we began to explore its implications for the strategy of research on schizophrenia; it pointed us in some new directions. First of all, it brought home to us the potential value of studying mildly-affected and clinically asymptomatic members of the families of schizophrenic probands. Chapman \& Chapman (1973) coined the term 'generalized deficit' to refer to the multiplicity of impairments of schizophrenic patients on nearly all psychological tasks. All severe disorders, as they progress, gradually become aetiological factors in their own right, causing general systemic malfunctions that may have little to do with the original causes of the disease. This process is characteristic of physical as well as mental disorders. Ultimately patients die, not from the disease which began their decline, but from a cascade of failures in organ systems, more alike than unique among patients whose disease processes started quite differently.

Generalized deficit has stood in the way of identifying specific and aetiologically relevant abnormalities in schizophrenia, and a great deal of effort has been wasted studying deficits that are only secondary consequences of the disease. If the genetic and biological factors leading to schizophrenia can also manifest themselves in subclinical or non-pathological forms, the opportunity arises to study those factors without the interference of the massive deficits in attention, motivation and task comprehension that are present in severe schizophrenia.

Mildly-affected and symptom-free family members have been a neglected resource for psychobiological investigation. Until now, in our own research we have sought instead to study the worst affected patients and the families with the highest concentration of illness. These 'high density' families, however, are not only rare, they are often uncooperative. Paradoxically, the social withdrawal, suspiciousness and interpersonal incompetence that comprise the schizophrenic syndrome make these interesting families the hardest to reach. The technique which we have now adopted is much more practical than the search for high density families. A premium is placed, not on finding families of a particular type, but on a thorough study of each of the relatives, including those who seem well.

The two manifest traits that have been coupled in our first application of the latent structure model, smooth pursuit tracking and schizophrenia, are very different from each other; it is not evident why they are related. We suspect that this is the general rule. Why in neurofibromatosis are 'café-au-lait' spots on the skin and multiple subcutaneous tumours related? For that matter, why in phenylketonuria are mental retardation and light pigmentation related? It is to be expected that an explanation of these couplings between disparate traits will be found in the course of time, but they must not be rejected at the outset just because they are not understood.

In our view, the apparent incomparability of the manifest traits that seem to be coupled, either within affected individuals or between members of the same family, is not an obstacle to research, but a powerful heuristic clue that should be pursued. As in navigation, fixing on several landmarks helps to triangulate one's position, so in the search for causes the attempt to make sense of divergent manifestations may point us towards an aetiological process. A cell type in the brain common to 
the several manifestations; a shared neurotransmitter; a common developmental epoch; vascular supply from the same arterial bed: any of these, and more could be explanations suggested by pondering the diverse manifestations of a latent trait.

This is a time-honoured technique in clinical neurology. The patient who complains of diverse and apparently unrelated symptoms is easier to diagnose than the one with a single symptom or sign. Certainly, the neurologist's ability to combine multiple manifestations is founded on extensive knowledge of the anatomy and physiology of the nervous system. In the same way, the more basic knowledge we have about the brain, the more sense we shall be able to make of divergent manifest traits that run in families. An excellent example of this kind of reasoning is the hypothesis of Geschwind \& Behan (1982) relating learning disorders, autoimmune disease and left-handedness to foetal testosterone as an underlying latent trait.

In approaching family members from the perspective of latent trait analysis, it is first of all necessary to go beyond the nosological attitude which has become predominant in modern psychiatry. Today's well-trained psychiatric interviewer examines members of the family with the mental set that they either have or do not have the diagnostic entity ascribed to the proband; or perhaps that they may have some other psychiatric syndrome listed in the diagnostic manual. This attitude would be acceptable if we had reason to believe that the diagnostic categories recognized, say by DSM-III or the ICD, correspond to distinct aetiological processes. Unfortunately for this method, there is no reason to assume that they do correspond, and abundant reason to expect that they do not. Therefore, while diagnostic categories may be useful for epidemiology, it is harmful to enforce them for genetic analysis when they have not been shown to be biologically well founded. Not only may variance be increased by incorporating several distinct illnesses within one category, but psychobiological phenomena that are genuinely important may be dismissed because they do not seem to respect diagnostic boundaries that are actually artificial. Most important, the nosological mental set is likely to blind the interviewer to relevant phenomena that are never studied simply because they are never recorded.

A technique of family interviewing is needed which maximizes qualitative information about disorders not obviously related to the disease of the proband; about subclinical manifestations; and about a broad spectrum of subtle cognitive, affective, and personality variables which may potentially be components of the same latent structure. This kind of interview is likely to be much more revealing than epidemiologically-based methods which simply assign individuals to pre-existing diagnostic categories.

The design of a 'latent structure' family interview is not a simple matter. The difficulty, of course, is that without a comprehensive theory of schizophrenia we do not know what to include in the interview; and without the data which such an interview might provide, we are not able to formulate the comprehensive theory. An orderly selection of traits must be made, and while unplanned and 'subjective' observations by interviewers should be encouraged and recorded, clinical intuition cannot substitute for reliable measures that have been worked out in advance.

To a large extent, the choice of topics to be included in a 'latent structure' family interview will depend on the interests and hypotheses of each research group that uses the method. We have thought about some general principles that can guide the choices that have to be made. It is likely that a top-down approach, which works its way gradually towards biochemical and genetic levels will be the most fruitful. Great leaps from psychiatric to molecular phenomena have not been successful in the past, and are not likely to work in the future. Most markers studied in the past, like MAO, tried to bridge the gap from molecules to mental state in one jump, and it would have been pure luck if correlations so far-reaching had been found.

Similarly, behavioural scientists have tried to study aspects of perception and cosnition that might be involved in schizophrenic disorders, such as focused attention, inhibition $r f$ distracting stimuli, perceptual distortion and thinking peculiarities. But many of the tests that 1 've been employed to measure those processes fail to isolate the specific components of performance that are relevant to schizophrenia. They have nevertheless been widely employed because of their availability and familiarity. Their yield has understandably been negligible. 
In the initial stages of genetic latent structure analysis, phenomena should be explored which point, Janus-like, in two directions at once: they should be tied to the psychology of schizophrenia, and at the same time related to known processes in the brain.

The eye movement dysfunctions have been explored in that way. From the psychological point of view, it was clear that a failure in voluntary attention was not involved in producing the tracking abnormalities, since all patients were trying to perform the task, and tests of more difficult and prolonged saccadic tracking showed no gross dysfunctions. Furthermore, manipulations of the target which engaged the viewer's attention more intensely had only minimal effects in reducing the saccadic intrusions. The pursuit abnormality was therefore assumed to involve involuntary or passive attention. From the physiological point of view, the abnormality was assumed to be located in higher brain centres because experiments had shown that the eyes of schizophrenic patients can be made to move smoothly when they are driven by brain stem and vestibular processes, as in the vestibulo-ocular reflex.

In principle, the prospects for research on schizophrenia should be quite bright. The powerful new imaging devices for brain function in vivo, the generally increased precision and subtlety of cognitive, affective, and psychophysical measurement and, above all, the great increase in knowledge of the brain in the last decade augur well for progress.

At the present time, however, it must be admitted that progress in schizophrenia research has paused on a plateau. The principal cause is not lack of funds but shortage of scientists with hopeful new leads; lack of funds is more a consequence than a cause. We believe that the principles we have presented, derived from the genetic latent structure model, can bring about a rejuvenation of psychobiological research on schizophrenia.

STEVEN MATTHYSSE AND PHILIP S. HOLZMAN

\section{REFERENCES}

Chapman, L. J. \& Chapman, J. P. (1973). Problems in the measurement of cognitive deficit. Psychological Bulletin 79, $380-385$.

Geschwind, N. \& Behan, P. (1982). Left-handedness: association with immune disease, migraine and developmental learning disorder. Proceedings of the National Academy of Science (USA) 79, 5097-5100.

Holzman, P. S., Kringlen, E., Levy, D. L., Proctor, L. R. \& Haberman, S. J. (1977). Abnormal pursuit eye movements in schizophrenia: evidence for a genetic marker. Archives of General Psychiatry 34, 802-805.
Holzman, P. S., Kringlen, E., Levy, D. L. \& Haberman, S. J. (1980). Deviant eye tracking in twins discordant for psychosis: a replication. Archives of General Psychiatry 37, 627-631.

Holzman, P. S. (1985). Eye movement dysfunctions in psychosis. International Review of Neurobiology 27, 179-205.

Marie, P. (1893). Hereditary cerebellar ataxia. Translated (1977) in Neurological Classics in Modern Translation (ed. D. A. Rottenburg and F.H. Hochberg), pp. 208-217. Hafner: New York.

Matthysse, S., Holzman, P. S. \& Lange, K. (1986). The genetic transmission of schizophrenia: application of Mendelian latent structure analysis to eye tracking dysfunctions in schizophrenia and affective disorder. Journal of Psychiatric Research (in the press). 